\title{
EFFECT OF SURFACE CONDITION ON THE TRANSPORT PROPERTIES
}

OF TUNGSTEN

D. R. Baer and D. K. Wagner

Laboratory of Atomic and Solld State Physics

Corne11 University

Ithaca, New York 14850

February 1973

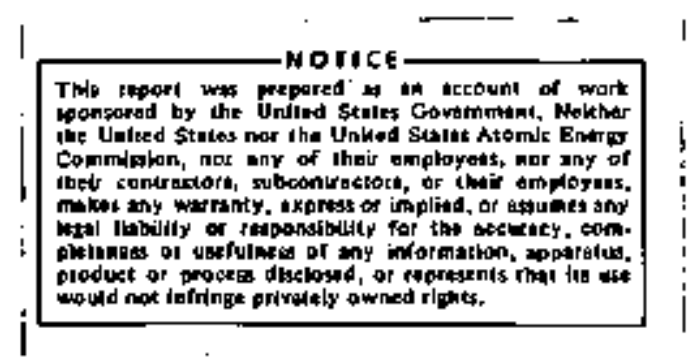




\section{DISCLAIMER}

This report was prepared as an account of work sponsored by an agency of the United States Government. Neither the United States Government nor any agency Thereot, nor any of their employees, makes any warranty, express or implied, or assumes any legal liability or responsibility for the accuracy, completeness, or usefulness of any information, apparatus, product, or process disclosed, or represents that its use would not infringe privately owned rights. Reference herein to any specific commercial product, process, or service by trade name, trademark, manufacturer, or otherwise does not necessarily constitute or imply its endorsement, recommendation, or favoring by the United States Government or any agency thereof. The views and opinions of authors expressed herein do not necessarlly state or reflect those of the United States Government or any agency thereof. 


\section{DISCLAIMER}

Portions of this document may be illegible in electronic image products. Images are produced from the best available original document. 
Effect of Surface condition on the Transport Properties of Tungsten ${ }^{*}$

D. R. Baer and D. K. Wagner

Laboratory of Atomic and Solid State Physics Corne11 University

Ithaca, New York 14850

\section{Abstract}

Measurements are presented of the low-temperature electrical and thermal resistivities, $\rho$ and $w$, of several higb-purity, single-crystal tungsten rods of various orientations for which the electron mean-free path $\&$ is larger than the sample diameter d. For the highest purity samples oxiented with a [110] direction parallel to the rod axis, we observe several effects of surface condition on the electron transport properties. Firstly, both the electrical and thermal resistivities are increased substantia11y by sandblasting or electroetching the surface, and reduced correspondingly by electropolishing the surface. Secondly, the temperature dependent part of the electrical resistivity $\left[\rho-\rho_{0}\right]$ is reduced by sandblasting or electroetching the surface, and increased by electropolishing the surface. The corresponding effect is not observed for the thermal resistivity. Thirdly, the Lorenz number extrapolated to $T=0 \mathrm{~K}, \mathrm{~L}(\mathrm{~T}-0)=(\rho / W T)_{0}$, is found to equal the standard 
$-2-$

value $\mathrm{L}_{\mathrm{o}}=2.445 \times 10^{-8} \mathrm{~W} \Omega / \mathrm{K}^{2}$ for the sandblasted or electroetched surface, but is reduced significantly (up to $18 \%$ ) below this value for the electropolished surface. In the latter case, application of a longitudinal magnetic field strong enough to prevent many of the conduction electrons from striking the surface is observed to increase $L(T \rightarrow 0)$ to $L_{0}$, confirming that this is, indeed, a surface effect. 


\section{Introduction}

It is well known that when the mean-free path of conduction electrons in a metal becomes comparable to specimen size, the surfaces can have an inportant effect on the transport properties. These size effects are conveniently divided into two regimes. The first is the classical size-effect regime in which the electron mean-free path $\ell$ is larger than the smallest specimen dimension d, but much larger than the electron wavelength $\lambda$. The second is the quantum size-effect regime in which $d$ is comparable to $\lambda$. This paper deals with classical size effects, which include: the enhancement of resistivity with decreasing specimen size, the change in temperature dependence of the resistivity with specimen size, and the decrease of the longitudinal magnetoresistance with increasing magnetic field. The literature on these effects is very extensive and has been reviewed recently by chambers 1 , Brändli and Olsen ${ }^{2}$, and Larson ${ }^{3}$.

The usual theoretical treatinents of classical size effects are semiclassical treatments based on the Boltzmann equation. Within this framework the electron wavefunctions are assumed to be essentially unperturbed by the presence of the surfaces, so that the surfaces act only to scatter the incident electrons. The nature of this scattering enters the theory as a boundary 
condition on the solution of the Boltzmann equation. Although simple in principle, a realistic solution of this equation is generaliy difficult in practice for two reasons. First, the detailed nature of the surface scattering. is not known, and second, even if it were, the problear of solving the Boltzmann equation for a given bulk scattering mechanism and Fermi surface would be exceedingly complicated except under special circumstances. Thus, it has been customary to assume that the surface scattering is either diffuse or specular or a mixture of each. Diffuse scattering refers to an event in which the incident electron has equal probability of being scattered into any state which carries the electron into the metal. Specular scattering, on the other hand, refers to an, event in which the component of wavevector parallel to the surface is conserved. (Inklapp processes, which are determined by the translational symmetrtes of the surface, are also possible.)

It has been customary, since the original work of Fuchs, 4 to describe the relative anounts of diffuse and specular scattering by the specularity parameter $p$ with $p=0$ corresponding to completely diffuse scattering and $p=I$ to specular scattering. Even with this simplification of the surface scattering, it usually has been necessary to assune that the bulk scattering can be described by a relaxation time and 
that the Fermi surface is spherical, to make the solution of the Boltzmann equation feasible.

The results of many size-effect experiments have supported the view that for metals the surface scattexing is mainly diffuse. ${ }^{1}$ However, there is evidence that specular scattering can be important. Studies of the electrical resistivity of thin films and whiskers ${ }^{3}$ and of the optical reflectivity of thin films 5 have strongly supported the existence of specular scattering under certain conditions. The most direct evidence is provided by the observation of thagnetic surface states ${ }^{6}$; these states could not exist if the surface scattering were entirely diffuse. However, in several investigations $5,7,8$ negative values of $p$ have been obtained, suggesting that under some circumstances the description of scattering at the surface in terms of the specularity parameter $p$ may be too great a simplification.

We have undertaken this study to explore in greater detail the effect of surface scattering on electron transport properties. 9 Since one expects the extremes of diffuse and specular scattering to correspond to very rough and very smooth surfaces, respectively (a more quantitative criterion for smoothness will be given later), we were led to investigate the effects of these two extremes of surface condition on the electrical resistivity, the thermal resistivity, the Lorenz number, and 
the longitudinal magnetoresistance of tungsten single crystals. Tungsten was chosen because it could be obtained in oriented single-crystal rods of high purity and with polished surfaces. Furthermore, previous measurements on tungsten ${ }^{10}$ indicated that the transport properties could be influenced by the condition of the surface.

There has been very little previous investigation of the effect of surface condition on transport properties. In fact, on at least one occasion when the surface condition was deliberately changed during the course of a size-effect experiment, it was reported that no concomitant change in the resistivity had occurred $^{11}$. However, we note that Schwarz ${ }^{12}$ has reported a change in the resistivity of a sample of cadnitum after etching the electropolished surface. Bennett et al. ${ }^{5}$ have studied the optical reflectivity of thin films of silver of known surface roughness and have concluded that for roughness less than $45 \dot{A}$ nms the scattering is predominately specular, while for greater degrees of roughness the scattering is predominately diffuse. Finally, Koch and Murray ${ }^{13}$ have investigated the effect of surface condition on magnetic surface states. 
II. Experimenta1 Details

Measurements were made on variously oriented singlecrystal tungsten rods ranging from 0.13 mm to 3 mu in diameter. These included measurements of the longitudinal magnetoresistance (LMR) at $4.2 \mathrm{~K}$ and the electrical and thermal reststivities, $P$ and $W$, as a function of temperature from 1.5 to $5.5 \mathrm{~K}$. The LMR measurements were useful primarily in indicating the amount of surface scattering present. The resistivity data, while also of considerable intrinsic interest, were used to calculate the Lorenz number, $L=\rho / W T$, as a function of temperature. The value of the Lorenz number extrapolated to zero temperature $L(T-0)$ was also of interest and was computed from the extrapolated values of $\rho$ and WT. For one sample the longitudinal thermal magnetoresistance was measured in addition to the electrical magnetoresistance. The surfaces of several of the samples were altered by plating, etching, sandblasting, and electropolisbing, and the effects of the surface condition on the above measurements were noted.

The IMR measurements were made in a superconducting solenoid whose field was homogeneous to about $0.1 \%$ over the sample volume. The samples were aligned in the field to about $1.5^{\circ}$; the alignnent was not cxitical for measurements below about $500 \mathrm{G}$, but at higher fields the LMR normally in reased with 
field at a rate that depended strongly on sample alignment. This was presumably due to a sma11 amixture of the larger transverse component of magnetoresistance, which increases quadratically with magnetic field.

The possible existence of anomalous LMR behavior due to current jetting ${ }^{14,15}$ was investigated by adding two extra voltage leads to the sample, so that the voltage could be measured across three sections of the sample. We found that the measured LMR was qualitatively the same across all three sections and that the measured voltage across two individual sections added to give the measured voltage across both sections together. Therefore, we could find no evidence that current jetting was playing any significant role in these measurements. The electrical and thermal resistivities were measured in the manner described by Wagner et a1. ${ }^{10}$. As tuch care as possible was taken to avoid strain on the smaller samples, although some was unavoidable and its effects were seen on the smallest sample. The precision of the measurements was essentially the same as given in Ref. 10 , being about $2 \%$ for the electrical measurements and $1 \%$ for the thermal measurements. We have attempted to observe the effects of our treatment on the tungsten surface by means of an optical microscope and a scanning electron microscope (SEM). Table I lists observed surface characteristics as viewed by the naked eye, 
by the optical microscope, and by the SEM. The resolution of the scanning electron microscope was slightly better than $300 \mathrm{~h}$. One should note, however, that the scale of surface roughness that is important for determining characteristics of surface scattering is probably at least an order of magnitude smaIler. Nonetheless, the SEM pictures, as shown in Figs, 1 and 2, are useful in showing the drastic differences In the sample surfaces produced by our treatments of the surface. The characteristics of each sample with the surface in a given condition are listed in Table II. It is important to note that some of the treatments of the surface were incomplete. For example, the $\mathrm{H}_{2} \mathrm{O}_{2}$ etch attacked specific planes on the sample surface and left a larger fraction of the surface unaffected. In these cases we have denoted the state of the surface as "selectively etched." As one would expect, these samples exhibited characteristics that were intermiediate between those exhibited when the surface was polished and those exhibited when the surface was uniformily etched. 
III. Experimental Results

One problem inherent in de size-effect studies is the determination of the relative purities of different samples of varying size. For bulk samples the residual resistance ratio, $I_{R}=\rho(299 \mathrm{~K}) / \rho(0 \mathrm{~K})$, is a good indicator of purity. However, when the electron mean-free path is size-limited, $r_{R}$ is affected by both bulk impurity scattering and surface scattering and is no longer a useful indicator of purity. Several methods ${ }^{16-18}$ have been used to surmount this difficulty, but none of them is completely satisfactory. Perhaps the simplest is that suggested by Berthe1. 17

According to this method, the quantity $\mathbf{r}_{\mathrm{R}} / \mathrm{d}$ is used, rather than $\mathbf{r}_{\mathrm{R}}$, to characterize purity in the regime $\ell \geq \mathrm{d}$. This approach can be illustrated by assuning that the resistivity is given by the Nordheim expression, which can be written

$$
\text { , } \mathbf{r}_{\mathrm{R}} / \mathrm{d}=\left(\mathrm{r}_{\mathrm{R}} / \ell\right)_{\text {bulk }} \frac{\ell}{\ell+\mathrm{d}} \cdot(\mathrm{p}=0)
$$

Since $\left(I_{R} / \ell\right)_{\text {bulk }}$ is a constant of the material in this model, it is seen that for $l \geq d, r_{R} / d$ depends much more weakly on d than does $r_{R^{\prime}}$ Also for a given value of $d, r_{R} / d$ is an increasing function of $\ell$ which saturates at the value $\left(r_{R} / \ell\right)_{b u l k}$ as $\ell$ becomes much larger than d. For these reasons we have found the quantity $r_{R} / d$ to be a useful although relatively crude indicator of sample purity. 
We would like to emphasize that it is not useful to attempt to refine this method until the nature of the scattering at the surface and the actual Fermi surface are taken into account. In support of this we note the following: According to Eq. (I) the maximum value of $r_{R} / d$ is $\left(r_{R} / \ell\right)_{b u 1 k}$, which is given by

$$
\left(r_{R} / 2\right)_{\text {butk }}=\rho(299 \mathrm{~K})\left[\frac{e^{2}}{2 \pi^{3} \mathrm{~h}} \mathrm{~s}_{\mathrm{F}}\right],
$$

where $S_{F}$ is the total Fermi surface area and $e$ is the electronic charge. For tungsten ${ }^{19},\left(r_{\mathrm{R}} / l\right)_{\text {bulk }}$ has the value of $58,000 \mathrm{~nm}^{-1}$. Referring to Table II, which sumarizes the characteristics of the tungsten specimens, we note that for sample $W-16$, orlented with a [111] direction para1le1 to the rod axis, the measured value of $r_{R} / d$ is $110,000 \min ^{-1}$. This is nearly twice the maximum value predicted by Eq. (I). One might first suppose that this discrepancy could be resolved by asstming that specular scattering is present $(p \neq 0)$. However, it is more likely that this large value of $r_{R} / d$ reflects the fact that, for the [111] orientation, the velocities of a large number of electrons on the octahedral hole sheet of the tungsten Fermi surface are nearly parallel to the rod axis. Since these electrons carry a disproportionate share of the transport current for $\ell \geq d$, it can be expected that the resistivity for this orientation would be anomalously low. 
We conclude, as does Larson ${ }^{3}$, that the Nordheim formula and similar, more exact treatments, 20,21 which assume an isotropic electron gas, can be misleading when applied to metals like tungsten which have a vexy anisotropic Fermi surface. Because of the strong influence of sample orientation on $r_{R} / d$, this quantity should be used as an indicator of sample purity only among specimens having the same orientation.

Better estimiates of the bulk electrical resistivity can be obtained by measuring the reduction of the resistivity in a longitudinal magnetic field. As the cyclotron radii of the carriers become sma1ler than the sample dlameter, the effect of surface scattering is greatly reduced and the resistivity approaches the value $1 t$ would have in a bulk sample in the same longitudinal magnetic fleld. When the bulk magnetoresistivity is taken into account, one can estimate the zero-fleld bulk resistivity, and hence the mean-fxee path $\ell$. By using the sample W-7 (the largest sample) as a gauge, we estimate that the bulk resistivity is increased approximately $20 \%$ by a longitudinal magnetic field. Using this value and the method outlined above, we estimate the mean-free path of sample W-12 to be about $1.8 \mathrm{~mm}$.

Among samples of similar size and the same orientation, there appear to be two principal types of LMR behavior as shown in Fig. 3. The first (marked I on the graph) consists 
of a decrease (occasiona1ly preceded by a sma11 increase)

in the resistivity with increasing field, the minimum resistivity of the sample being $50 \%$ to $60 \%$ of the zero-field value. The second (marked II on the graph) consists of an increase in the resistivity of about $10 \%$ and then a decrease to approximately the zero-field value at higher fields. of the samples having a [110] orientation, those exhibiting the first type of LMR behavior appear to be the purest, since these samples have the largest values of $r_{R} / d$. The two different types of behavior can be understood qualitatively by a very simple model which is outlined in the next section. As one would expect, both behaviors show that the smaller the sample, the slower the decrease in resistivity with increasing field. Figure 4 focuses on the LMR of sample $W-12$ for various surface conditions and demonstrates that the zero-fleld electrical resistivity can be changed reversibly by altering the condition of the sample surface. The LMR is shown for the initial polished state, after etching, after repolishing, and after sandblasting. By roughening the surface, the zerofield electrical resistivity is increased, while by repolishing the surface the zero-field resistivity is decreased. ${ }^{22}$ In a11 cases, however, the resistivity approaches essentially the sane value as the magnetic field increases. Because the fraction of the electrons striking the surface decreases with 
increasing longitudinal magnetic field, this observation suggests that the bulk properties of the specimens have not been altered by our treatment of the sample surface.

of the samples measured in this study and in the previous study by Wagner, et a1. ${ }^{10}$, samples $w-8$ and $w-12$ exhibit characteristics that clearly set them apart from the others, The temperature dependence of the electrical resistivity of these two samples is larger than normal, as can be seen by referring to Fig. 5 and Ref. 10. Moreover, their Lorenz numbers extrapolated to zero temperature $\mathrm{L}(\mathrm{T} \rightarrow 0)$ are substantially lower than $L_{0}$ when their surfaces are in the polished condition. With the surfaces in the etched condition, these samples exhibit the normal behavior with $L(T \rightarrow 0)$ essentially equal to $\mathrm{L}_{0^{*}}$. From the behavior of the LMR of these samples (see Fig. 3) and their large values of $r_{R} / d$, these two specimens appear to be the purest. Because of their anomalous behavior, most of our attention has been devoted to these samples, particularly to $\mathrm{W}-12$.

It is important to mention that samples $W-8$ and $W-12$ are essentially no different than the other samples in one respect: The temperature dependence of WT for these two samples is nearly identical to that of the other samples, as shown in Fig. 6 and Ref. 10.23

Figure 7 shows the temperature dependence of the electrical 
resistivity of sample W-12 for various conditions of the surface. From this figure it can be seen that the magnitude and temperature dependence of the resistivity change with surface condition. Roughening the surface increases the electrical resistivity while polishing the surface decreases it as observed previously in Fig. 4. The thermal data, seen in Fig. 8, also shov an increase after the surface is roughened and a decrease after the surface is repolished. A careful look at the temperature dependences of the electrical and themal resistivities shows, however, some differences in the effect of the surface on electrical and thermal conduction. It is observed from Fig. 9 that sandblasting the surface decreases the temperature dependence of the electrical resistivity relative to the Initial polished state, while repolishing the surface increases the temperature dependence to nearly that of the original polished sample. In all cases the temperature dependence of the electrical resistivity of this sample is greater than that of a typical 'bu1k' sample. In contrast, Flg. 10 shows the temperature dependent part of WT, and it is noted that surface condition plays little, if any, role in the temperature dependence of this quantity. 24

The Lorenz number, $\mathrm{L}=\rho / \mathrm{WT}$, is also found to depend upon the surface condition of the sample. Figure 11 shows the Lorenz number of sample W-12 as a function of temperature for 
various surface conditions. It is seen from the figure that the Lorenz number is depressed by electropolishing the surface and is increased by sandblasting the surface. In particular, the Lorenz number extrapolated to zero temperature $L(T \rightarrow 0)$ is depressed below $\mathrm{L}_{\mathrm{o}}$ for the polished surface and is increased to nearly $I_{0}$ for the sandblasted surface.

Further evidence that the condition of the surface is responsible for this effect is provided by measurements of the electrical and thermal resistivities of the repolished sample (W-12G) in a longitudinal magnetic field strong enough to remove most surface scattering. The results of this measurement are plotted in Fig. 12. From the graph it can be seen that at a field of $480 \mathrm{G}, \mathrm{L}(\mathrm{T} \rightarrow 0)$ has a value of nearly $\mathrm{L}_{\mathrm{o}}$, while at zero field it approaches a value about $10 \%$ 1ower. Since it is we11 known for a bulk metal that the Lorenz number will equal $\mathrm{L}_{\mathrm{o}}$ if scattering is elastic (this is also valid in a longitudinal magnetic field), these results demonstrate directly that the observed deviations from the Wiedemann-Franz law must be ascribed to the polished condition of the surface. The data also show a decrease in the thermal magnetoresistivity with increasing field similar to that observed in the electrical case.

We have also experimented'briefly with another treatment of the surface. By plating the surface of sample $W-12 G$, both the 
electrical and themal resistivities are increased proportionally, so that the Loxenz number is not significantly changed. Apparently, the increased surface scattering due to the gold plating affects the thermal conduction to the same degree as the electrical conduction.

The dependence of the Lorenz number on surface condition exhibited by sample $W-12$ contrasts with that exhibited by sample $W-15$, a sma1ler sample with the same orientation and a slightly lower value of $r_{R} / d$. The Lorenz number of sample W-15 shows no significant dependence on surface condition, although the temperature dependence of the electrical resistivity is decreased by sandblasting just as for sample $\mathrm{W}-12$. One should note that the temperature dependence of $p$ and WT for sample W-15A is laxger than that of more 'typical' samples, such as samples W-4 or W-7 (see Figs, 5 and 6 ). We believe that this is due to strain and have observed similar, more exaggerated behavior in a small, badly strained specimen (W-14). This illustrates one of the difficulttes in making meaningful measurements on very fine specimens.

We have observed aging effects in samples $\mathrm{W}-8$ and $\mathrm{W}-12$. Such effects have also been observed by Walsh ${ }^{25}$ in tungsten. Over a period of six months, during which the samples were kept at room temperature, the electrical resistivity of $\mathrm{w}-12$ in the inftial polished condition was observed to increase 
about $15 \%$ while the surface condition was not deliberately altered. We also made new measurements on sample $\mathrm{W}-8 \mathrm{~B}$ a year and a half after the initial measurements had been made. ${ }^{9}$ In the original measurement $\mathrm{L}(\mathrm{T} \rightarrow 0)$ was found to be essentially equal to $\mathrm{L}_{0}$, while in our subsequent measurement on the sample we found that it had decreased well below $\mathrm{L}_{\mathrm{o}}$. Sandblasting the surface of this sample produced only very slight changes in the electrical and thermal resistivities, in marked contrast to results on other samples. This suggests that a surface layer may have formed on the sample. After removing $0.3 \mathrm{~mm}$ from the diameter of $\mathrm{W}-8 \mathrm{~B}$ by electropolishing, this sample was observed to behave as it did orlginally. We have looked for aging effects in some of our other samples, but have not found any nearly as large as those shown by W-8 or $\mathrm{W}-12$. 
IV. Discussion

The longitudinal magnetoresistance measurements reported here are similar to those that have been made in other metals. ${ }^{2}$ For samples of the same size and orientation we find two characteristic types of LMR behavior determined largely by sample purity. This behavior can be explained with the modified Kohler's rule proposed by $01 s e n, 26$ or perhaps more simply by assuring that the LMR consists of three components: a constant term corresponding to the zero-field value of the resistivity, a negative term due to the decreasing effect of surface scattering as the magnetic field is increased, and a positive term corresponding to the bulk magnetoresistance.

If one uses the calculation by Chambers ${ }^{27}$ for the sizeeffect tem and assmes that the bulk LMR saturates at a field inversely related to the mean-free path, one can roughly account for the two behaviors observed. As $l$ is varied from $\ell<d$ to $l>d$, the shape of the LMR curve changes slowly from a sma1l rise followed by a small decrease (curve II) to a very small rise followed by a large decrease (curve $\mathrm{I}$ ). The character of the LMR behavior depends, therefore, on sample purity. This analysis is consistent with earlier comments in this paper that samples $W-8$ and $w-12$ are purer than other samples and therefore have the longest mean-free paths. This simple model ignores orientation effects. 
The LMR measurements have established that significant surface scattering is present in the samples, and also have indicated to some extent the relative amounts of such scattering anong the samples. These measurements also have been useful in establishing that the surface treatment changes the zerofield resistivity, while not significantly changing the bulk resistivity. Thus, we conclude that our treatnent of the surface has not significantly altered the interior of the sample. Our observation that the resistivity can be appreciably affected by treatment of the surface contrasts sharply with the results of startsev et al. ${ }^{11}$, who observed no dependence on surface condition in their size-effect study on tungsten. The dependence of the resistivity on surface condition implies that by changing the surface one has changed the nature of the surface scattering. In the simplest model, one might assume that the proportion of diffuse and specular scattering has been changed or, for example, that the specularity parameter $p$ is decreased by roughening the surface. Way and $\mathrm{Kao}^{28}$ have calculated the LMR of thin films for different values of $p$, and their results could be used to infer the existence of specular scattering in our samples. However, even if we assme that these results would be similar to those for a wire, one must still keep in mind that there are inportant differences in the nature of specular scattering for a metal 
with a very anisotropic Fermi surface (such as tungsten) and for the free electron gas assumed in the calculations of way and $\mathrm{Kao}^{28}$. For a metal with an anisotropic Fermi surface, the angle of incidence will not necessarily equal the angle of reflection, and furthermore, an electron may scatter to more than one location on the Fermi surface 29,30 . If one includes unklapp processes, the number of such possibilities can become very much greater ${ }^{31}$, especially if the surface does not lie in a major crystallographic plane.

The separation of surface scattering into a specular component and a diffuse (or random) component is probably a rather drastic simplification of the problem in most situations. R. F. Greene ${ }^{29}$ has formulated in general terms the surface boundary condition for electron-surface scattering using current conservation at the surface in the tsual semiclassical theory. The resulting boundary condition is equivatent to the assumption of a specularity parameter $p$ only in special cases, and even for these cases $\mathrm{p}$ will generally depend upon the electron distribution function. Thus, $p$ will be different for different transport processes. However, the general boundary condition is difficult to apply, so it has usually proven convenient to asstme that a specularity parameter exists, keeping in mind the possible limitations.

One obvious consideration is that the nature of the surface 
scattering should depend upon the angle at which the electron collides with the surface, and various workers have investigated theoretically the dependence of $\mathrm{p}$ upon the scattering angle. ${ }^{3,31}$ In particular, soffer ${ }^{32}$ has shown by simple diffraction considerations for a surface with uncorrelated irregularities, that the specularity parameter $p$ is given by

$$
p(\cos \theta)=\exp \left[-(4 \pi h \cos \theta / \lambda)^{2}\right],
$$

where $\theta$ is the angle of incidence, $h$ is the rmis roughness, and $\lambda$ is the deBroglie wavelength of the electrons. From this formula one can see that $p$ is appreciable only for glancing angles of incidence even if the surface is atomically smooth. For example, if $\mathrm{h}=\lambda$ (in a metal this corresponds to an atomically snooth surface), then $p>\frac{1}{e}$ only for angles of incidence greater than $85^{\circ}$. In most size-effect phenomena, the electrons travelling nearly parallel to the surface (with large angles of incidence) play an important role. In the dc size effect, $\cos \theta$ is roughly $\mathrm{d} / \ell$ or less for the electrons which transport the bulk of the current. In the anomalous skin effect, the important electrons are the effective ones for which $\cos \theta<\delta / \ell$, where $\delta$ is the skin depth. For magnetlc surface states, $\cos \theta$ is about $2 \sqrt{2 z_{1} / R}$ where $z_{1}$ is the maximum depth achieved by the ground state trajectory and $R$ is its radius of curvature. In the latter case $\cos \theta$ is typically 0.01 , which corresponds 
to a value of $p$ of 0.99 for an atomically smooth surface. In the case of dc transport, values of $d / \&$ apprectably less than unity can be obtained only in very fine specimens at low temperatures. Thus, it seems from these simple considerations that specular scattering should be completely dominant for magnetic surface states, but diffuse scattering should be predominant for de transport phenomena.

Greene $^{33}$ has argued that, for a degenerate system, $p$ might be greater than one would expect from simple diffraction considerations. His argument is based on the observation that for diffuse scattering the initial and final occupation factors must be included, while for specular scattering they need not be, since specular scattering is simply a way of constructing the standing wave states required by the presence of the boundary. Because quantitative calculations based on these arguments require detailed knowledge of transition probabilities, it is not known whether such a mechanism can produce the large value of $p$ necessary to make specular scattering in dc transport processes more plausible from a theo retical point of view.

In spite of the theoretical difficulties, there is considerable experimental evidence for specular scattering in de transport phenomena, as we discussed in the introduction. It is tempting to view many of our results in terms of specular 
and diffuse scattering, but we have had difficulty understanding them consistently in these terms, as we discuss later in this section.

Other experimenters have noted the effect of chemical coatings 8,34 . on the resistivity of metals. The surfaces of our samples were not ultra-clean, so such effects could be important. In general, the changes we observe in the resistivity due to changing the surface condition are larger than those produced by coatings. 34,35 A very simple view of the possible effects of chemisorbed atorns allows the electron density of the metal and the surface scattering to change. For a compensated metal, such as tungsten, a small change in the electron density would produce little change in the resistivity, but. would cause a larger change in the Hall voltage such as observed by Bast ${ }^{35}$. A change in surface scattering could increase the resistivity, and we may have observed this effect when the sample had aged and possibly when the sample was gold plated.

An increase in the temperature dependence of the electrical resistivity has been observed by many others in size-effect studies in several metals. ${ }^{2}$ Although these results are usually called deviations from Mattheissen's rule, ${ }^{36}$ Ehrlich $^{37}$ and Boughton and Neighbor ${ }^{38}$ point out that these increased temperature dependences are to be expected from a careful analysis of the conventional size-effect theory. The mechanism 
proposed by $01 \operatorname{sen}^{26}$ to explain the increased temperature dependence of the resistivity of small wires has been shown by Boughton and Neighbor ${ }^{38}$ to be largely unnecessary, but could still be present. Such a mechanism would, however, predict an increased temperature dependence for a roughened surface compared to a smooth surface, in contrast to our results. Although Boughton and Neighbor have not investigated the role of specular scattering in their model, a standard treatuent ascribing very little, if any, temperature dependence to the specularity parameter $p$ would give, for a sample with $p$ nearly equal to unity, a temperature dependence similar to that of a bulk sample. This would contrast with our results, in which the smoothest sample (that most likely to have large amounts of specular scattering) had the largest temperature dependence, while the roughest sample (that most likely to have large amounts of diffuse scattering) had a temperature dependence more nearly like that of a bulk sample. If, as others have suggested ${ }^{39}$, the specularity parameter has a large temperature dependence ( $p$ decreasing with increasing temperature), this could qualitatively explain our observations.

Another interesting aspect of the data is the observation that, although the temperature dependence of the electrical resistivity changes with surface condition, that of the thermal resistivity does not change. This is most certainly related 
to the fact that the electron mean-free path for thermal conduction is shorter than that for electrical conduction in the temperature range of our measurements. On this basis one expects that the thermal resistivity would be less sensitive than the electrical resistivity to the presence of the surfaces, and this is consistent with all our observations.

We now turn to a discussion of the Lorenz nuber neasurements. Normally in a bulk metal the Lorenz number is a good Indicator of the amount of smal1-angle inelastic scattering present. Such scattering (e.g. scattering by phonons at low temperatures) has the effect of reducing the Lorenz number $\mathrm{L}$ below the standard value $L_{o}=2.445 \times 10^{-8} \mathrm{w} / \mathrm{K}^{2}$. (Electronelectron scattering can also cause such behavior.) At even lower temperatures these processes become less important than the temperature-independent elastic processes, such as scattering by defects, impurities, etc, and $L$ returas to the value $\mathrm{L}_{0}$. However, this pattern is not followed by samples $\mathrm{W}-8$ and W-12 when their surfaces are in the electropolished state; their values of $L(T \rightarrow 0)$ are less than $L_{0}$, as discussed in Sec. III. The significance of this result depends upon two considerations, First, does the extrapolation to $\mathrm{T}=0 \mathrm{k}$ actually reflect the real situation or are the extrapolations misleading? Seond, if the extrapolations do reftect the true situation at $T=0 \mathrm{k}$, how can this result be explained? 
We cannot rule out the possibility that the extrapolations might be misleading. However, in order for the Lorenz number to approach $\mathrm{L}_{\mathrm{O}}$ at $\mathrm{T}=0 \mathrm{~K}$, the character of the temperature dependence of the resistivities would have to change dramatically below $1.5 \mathrm{~K}$. Such a change might be produced by the freezing out of some inelastic surface scattering mechanism, and would be of considerable interest for its own sake. clearly, measurements at lower temperatures would be helpful in clarifying this point.

However, if our extrapolations do reflect the true behavior of the Lorenz number, then this presents serious problems that are dfficult to resolve by conventional treatments of dc size effects. One might attempt to resolve the problem by postulating two different specularity parameters, one for electrical conduction and one for thermal conduction. Then the deviation of $\mathrm{L}(\mathrm{T} \rightarrow 0)$ from $\mathrm{L}_{0}$ would be attributable to the difference between the two parameters. However, this flexibility does not exist when all scattering mechanisms are elastic, and we show in an appendix that one expects $L(T \rightarrow 0)$ to equal $\mathrm{L}_{\mathrm{o}}$ for elastic bulk and surface scattering mechanisms. Consequently, we do not have a satisfactory explanation for these deviations from the law of Wiedemann and Franz. 


\section{Conclusion}

In this study we have shown that for very pure, singlecxystal tungsten rods, oriented with the rod axis parallel to a [110] direction, the condition of the surface can markedly affect the magnitudes of the electrical and thermal resistivities, the temperature dependence of the electrical resistivity, and the value of the Lorenz numer extrapolated to zem temperature $\mathrm{L}(\mathrm{T}-0)$. Furthermore, these effects are observed to be reversible -- that is, they can be restored by appropriate treatment of the surface. We believe this to be the first detailed study of the effects of surface condition on the de transport properties of a pure metal.

clearly, there is much wark left to be done on this subject. For example, future work in tungsten should deal with the role played by crystal orientation. An effort must be made to quantify surface roughness, and to prepare and maintain surfaces of known condition. The techniques developed in the field of surface physics should be extremely useful in this regard. It is possible that future systematic studies of the effects of surface condition on transport properties could be important in elucidating the interaction of conduction electrons with the metallic surface. 


\section{Acknowledgments}

We axe very grateful to $B$. Addis for zone refining and electropolishing the tungsten specimens used in this study. One of us (DRB) thanks $T$. N. Rhodin for providing helpfuI insight on aspects of surface physics during many discussions, and $R$. Bowers for guidance and consultation as thesis adviser. We are also grateful to B. W. Maxfield for several useful suggestions made during the course of this work and for continuing interest in this investigation. 
APPENDIX: Proof of the Validity of the Wledemann-Franz Law in the Classical Size-Effect Regime for Elastic Scattering.

Consider a cylindrical conductor oriented with the cylinder axis along the $z$ direction. We apply an electric field $\mathrm{E}_{z}$ or a thermal gradient $\nabla_{z} T$ tio the conductor which perturbs the equilibrium Fermi distribution function $f_{o}$ by the amount $e E_{z}{ }^{\top} E$ in the electrical case, and by $\left(-k_{B} \nabla_{z} T\right) \pi_{T}$ in the themal case (e is the electronic charge and $k_{B}$ is Boltzmann's constant). The quantities $\eta_{\mathrm{E}}$ and $\eta_{T}$ are determined by the Boltzmann equation, which can be written in each case as

$$
\vec{v} \cdot \vec{v}_{\mathbf{r}} \eta_{E}=\left(-\frac{\partial \hat{E}_{\mathrm{o}}}{\partial \varepsilon}\right) v_{z}+W\left(\eta_{E}\right)
$$

and

$$
\vec{v} \cdot \vec{\nabla}_{\mathbf{r}^{\eta} \eta_{T}}=\left(-\frac{\partial f}{\partial \varepsilon}\right) u v_{2}+w\left(\eta_{T}\right)
$$

where $\vec{\nabla}_{\mathbf{r}}$ is the transverse (radial) gradient operator, $u=(\varepsilon-\mu) / k_{B} r, E$ is the election energy, $\mu$ is the chemical potential, $\vec{v}$ is the electron velocity, and $W(\eta)$ is the collision integral which will be defined shortly. For simplicity we have considered only one band, but the following arguments can be readily generalized to include several bands. Both $\eta_{E}$. and $\eta_{T}$ are functions of wavevector $\vec{k}$ and radial position $x$. The boundary condition for scattering from the surface can be written in at least two equivalent ways. 39,40 The condition used by Greene is based on the requirement that the net current through a given point on the surface be zero. 
That condition is

$$
v_{n}\left(\vec{k}^{+}\right) \eta_{E}\left(\vec{k}^{+}\right)=-\int d \vec{k}^{-} v_{n}\left(\vec{k}^{-}\right) \eta_{E}\left(\vec{k}^{-}\right) w\left(\vec{k}, \vec{k}^{+}\right)
$$

for each point on the boundary and each state $\vec{k}+$. A stmilar condition exists for the thermal case with $\eta_{E}$ replaced by $\eta_{T}$. $v_{n}$ is the component of velocity normal to the surface, $\vec{k}^{-}$ represents the wavevector of a state with velocity incident on the surface, and $\overrightarrow{\mathrm{k}}^{+}$represents a state with velocity directed away from the surface. $w(\vec{k}, \vec{k}+)$ is the probabjlity for scattering at the surface from state $\vec{k}^{-}$to $\vec{k}^{+}$.

For bulk impurity scattering the collision integral can be written as

$$
W(\eta)=-\frac{1}{k_{B} T} \frac{1}{4 \pi^{3}} \int d \vec{k}^{\prime}\left(\psi-\psi^{\prime}\right) P\left(\vec{k}^{\prime}, \vec{k}^{\prime}\right),
$$

where $P\left(\vec{k}, \vec{k}^{\prime}\right)$ is the equilibrium transition rate between states $\vec{k}$ and $\vec{k}^{t}$ and is defined by $\eta=\left(-\frac{\partial f_{o}}{\partial \varepsilon}\right) \downarrow$. For elastic scattering $P\left(\vec{k}, \vec{k}^{\prime}\right)=\delta\left(\mathcal{E}-\mathcal{E}^{1}\right) P^{\prime}\left(\vec{k}, \vec{k}^{\prime}\right)$ and with this form for $P\left(\vec{k}, \vec{k}^{\prime}\right)$ it can be readily shown that

$$
\cdot w\left(u \eta_{E}\right)=u\left(\eta_{E}\right)
$$

Thus, if $\eta_{\mathrm{E}}$ is the solution to the first Boltzmann equation (A1), then $\eta_{T}=u \eta_{E}$ is a solution of the second (A2), provided the boundary condition (A3) is also met. We have assumed that $\vec{\nabla}_{r} u=0$, or equivalently that there is no radial variation in the chemical potential $\mu$ or temperature $T$; we believe this to 
be a sound assumption.

To show that the boundary condition is satisfied, we note that for elastic scattering $w\left(\vec{k}^{-}, \vec{k}^{+}\right)=\delta\left(\mathcal{C}^{+}-\varepsilon^{-}\right) w\left(\vec{k}, \vec{k}^{+}\right)$. Using this form for $w\left(\vec{k}, \vec{k}^{+}\right)$, it is easy to show that if $\eta_{E}$ satisfles the boundary condition for electrical conduction, $\eta_{T}=u \eta_{E}$ will satisfy the boundary condition for thermal conduction.

To compute the Lorenz number $\mathbf{L}$, one needs to calculate the electrical and thermal currents, or equivalently the electrical and thermal conductivity tensor elements $\sigma_{z z}$ and $x_{z z}$ which are given by

$$
\begin{aligned}
\sigma_{z z} & =\frac{e^{2}}{4 \pi} \int d \vec{k} v_{z} \eta_{E} \\
k_{z z} & =\frac{k_{B}^{2} T}{4 \pi} \int d \vec{k} u v_{z} \eta_{T} \\
& =\frac{k_{B}^{2} T}{4 \pi^{3}} \int d \overrightarrow{k u}^{2} v_{z} \eta_{E}
\end{aligned}
$$

By writing the integral over wavevector as an energy and surface integral in the usual manner one has

$$
\frac{\kappa_{z z}}{\sigma_{z z} T}=\left(\frac{k_{B}}{e}\right)^{2} \frac{\int_{-\infty}^{\infty} d u u^{2} \operatorname{sech}^{2} \frac{u}{2}}{\int_{-\infty}^{\infty} d u \operatorname{sech}^{2} \frac{u}{2}}=\frac{\pi^{2}}{3}\left(\frac{k_{B}}{e}\right)^{2}=L_{o}
$$

to order $\left(\frac{k_{B} T}{\mu}\right)^{2}$. This completes the proof. It is important to mention at this point that the measured Lorenz number is 
$\rho_{z z} / W_{z z} T$ and that this is equal to $x_{z z} / \sigma_{z z} T$ provided that one neglects themoelectric effects and provided that the cylinder axis is along an axis of twofold or higher symmetry. The latter condition is satisfied for all our specimens. 


\section{References}

* Work supported by the U. S. Atomic Energy Commlssion under Contract Number AT(11-1)-3150, Technjcal Report \$CO0-3150-11.

1. R. G. Chambers, in Physics of Metals: I Electrons, edited by J. M. Ziman (Cambridge Untversity Press, Cambridge, 1969), Chapter IV.

2. G. Brändli and J. L. Olsen, Mat. Sci. Eng. 4, 61 (1969).

3. D. C. Larson in Physics of Thin Films, Vol, 6, edited by M. H. Francombe and R. W. Hoffman (Academic Press, N.Y. 1971), p. 81

4. K. Fuchs, Proc. Cambridge Phil. Soc. 34, 100 (1938).

5. H. E. Bennett, J. M. Bennett, E. J. Ashley, and R. J, Motyka, Phys. Rev. 165, 755 (1968).

6. J. F. Koch, in Solid State Physics, Vol. I, Electrons in Metals, edited by J. F. Cochran and R. R. Haering (Gordon and Breach Science Publishers, Inc., N.Y. 1968), P. 253.

7. V. A. Marsocci and S. S. Shue, J. Appl. Phys. 42, 5047 (1971).

8. K. L. Chopra and M. R. Randlett, J. Appl. Phys. 38, 3144 (1967).

9. A preliminary report of the work presented in this paper was given at the 13 th International Conference on Low-Temperature Physics, Boulder, Colorado, August 1972.

10. D. K. Wagner, J. C. Garland and R. Bowers, Phys. Rev. B $\underline{3}$, 3141 (1971). 
11. v. Ye Startsev, N. V. Volkenshteyn, and G. Q. Nikitina, Fiz. Meta1. Metalloved $\underline{26}, 261$ (1968) [Phys. Met. Metallog. (USSR) 26, $76(1968)]$.

12. H. Schwarz, Phys. Kond. Mater. 9, 164 (1969).

13. J. F. Koch and T. E. Murray, Phys. Rev. 186, 722 (1969).

14. W. A. Reed, E. I. Blount, J. A. Marcus, and A. J. Arko, J. App1. Phys. 42, 5453 (1971).

15. J. A. Delaney and A. B. Pippard, Rep. Prog. Phys. 35, 677 (1972).

16. R. I. Balcombe and R. A: Barker, Ph11. Mag. 21, 533 (1970).

17. K. H. Berthel, Phys. Stat. Sol. $\underline{5}, 159$ (1964).

18. P. Cotti, Phys. Kond. Mater. $\underline{3}, 40$ (1964).

19. We have used the value of $S_{F}$ obtained from de Haas van Alphen tueasurements in tungsten by R. F. Girvan, A. V. Gold, and R. A. Phtllips, J. Phys. Chem. Sollds 29, 1485 (1968).

20. R. B. Dingle, Proc. Roy, Soc. (London) A 201, 545 (1950).

21. D. K. C. MacDonald and K. Sargenson, Proc. Roy. Soc. A 203, 223 (1950).

22. This behavior is distinct from the monotonic change in the resistivity caused by the reduction of sample diameter.

23. Samples that were strained such as sample W-15, showed an increased temperature dependence for both $P$ and $\mathrm{WT}$. 24. There was a slight change in the slope of WT vs. $\mathrm{T}^{2}$ as the sample size markedly decreased. 
25. W. M. Walsh in Solid State Physics, Vol. I: Electrons in Metals, edited by J. M. Ziman (Cambridge University Press, Cambridge 1969), p. 127.

26. J. L. Olsen, HeIv, Phys. Acta 31,713 (1958).

27. R. G. Chambers, Proc. Roy, Soc. A 202, 378 (1950).

28. Yuan-Shun Way and Yi-Han Kao, Phys, Rev. B , 2039 (1972).

29. R. F. Greene in Solid State Surface Science, Vo1. I, edited by M. Green (Marcel Dekker, N.Y. 1969), Chapter II.

30. A. P. Pippard, The Dynamics of Conduction Electrons (Gordon and Breach, N.Y. 1965), P. 46.

31. A. F. Andreev, Usp. Fiz. Nauk. 105, 113 (1971) [Sov, Phys.-Usp. 14, $609(1972)]$.

32. S. B. Soffer, J. App1. Phys. 38, 1710 (1967).

33. R. F. Greene, Phys. Rev, 141, 687 (1966); also see Refs. 2 and 3.

34. J. W. Geus, in Physical and Chemical Aspects of Adsorbents and Catalysts, edited by B. G. Linsen (Academic Press, London 1970), p. 529.

35. Z. Bast1, Thin Solid Films 10, 311 (1972).

36. J. Bass, Adv. Phys. 21, 431 (1972).

37. A. C. Ehrlich, J. Appl. Phys. 42, 2598 (1971).

38. R. I. Boughton and J. E. Neighbor, J. Low Temp. Phys. I, 241 (1972). 
39. Yu. P. Gaidukov and Ya Kadletsova, Zh. Eksp. Teor. Fiz. 57, 1167 (1969) [Sov. Phys. JETP 30, 637 (1970)].

40. J. M. Ziman, Electrons and Phonons (Oxford University Press, Oxford, 1960), p. 453 . 


\section{Figuxe Captions}

Fig. 1: Photograph of polished surface at $x 10,000$ on scanning electron microscope. Etch pits are used to help focus microscope.

Fig. 2: Photograph of sandblasted surface at $x$ 5,000 on scanning electron microscope.

Fig. 3: Longitudinal magnetoresistance as a function of magnetic field for various samples. A11 samples have a [110] orientation paralle1 to rod axis except $W-16$ and $W-17$, which have [111], and [100] orientations, respectively, All data were taken at $4.2 \mathrm{~K}$ except as otherwise indicated.

Fig. 4: Longitudinal magnetoresistivity as a function of magnetic field at $4.2 \mathrm{~K}$ for various conditions of sample surface.

Fig. 5: Varfation of the temperature dependent part $\left[\rho-\rho_{0}\right]$ of the electrical resistivity with temperature for various samples.

Fig. 6: Variation of temperature dependent part [WT - $\left.(\mathrm{WT})_{0}\right]$ of the themal resistivity muliplied by temperature for severa1 samples.

Fig. 7: Electrical resistivity $p$ as a function of temperature for various surface conditions. 
Fig. 8: Thermal resistivity multiplied by temperature WT as a function of temperature for various conditions of sample surface.

Fig. 9: Variation of the temperature dependent part [ $\rho$ - $\left.\rho_{0}\right]$ of the electrical resistivity with temperature for various conditions of sample surface compared to a typical butk sample.

Fig. 10: Temperature dependent part [WT - (WT) ${ }_{o}$ ] of the thema1 resistivity multiplied by temperature as a function of temperature for several surface conditions of sample W-12.

Fig. 11: The Lorenz number as a function of temperature. Fig. 12: Temperature dependence of the electrical and thermal resistivities of sample $W-12 G$ at zero field and in' a longltudinal magnetic fie1d. The circled and square data points were taken in the same experiment. The data points indicated by triangles were taken in a separate experiment. 
Table $?$ Surface characteristics of tungsten samples for different treatments

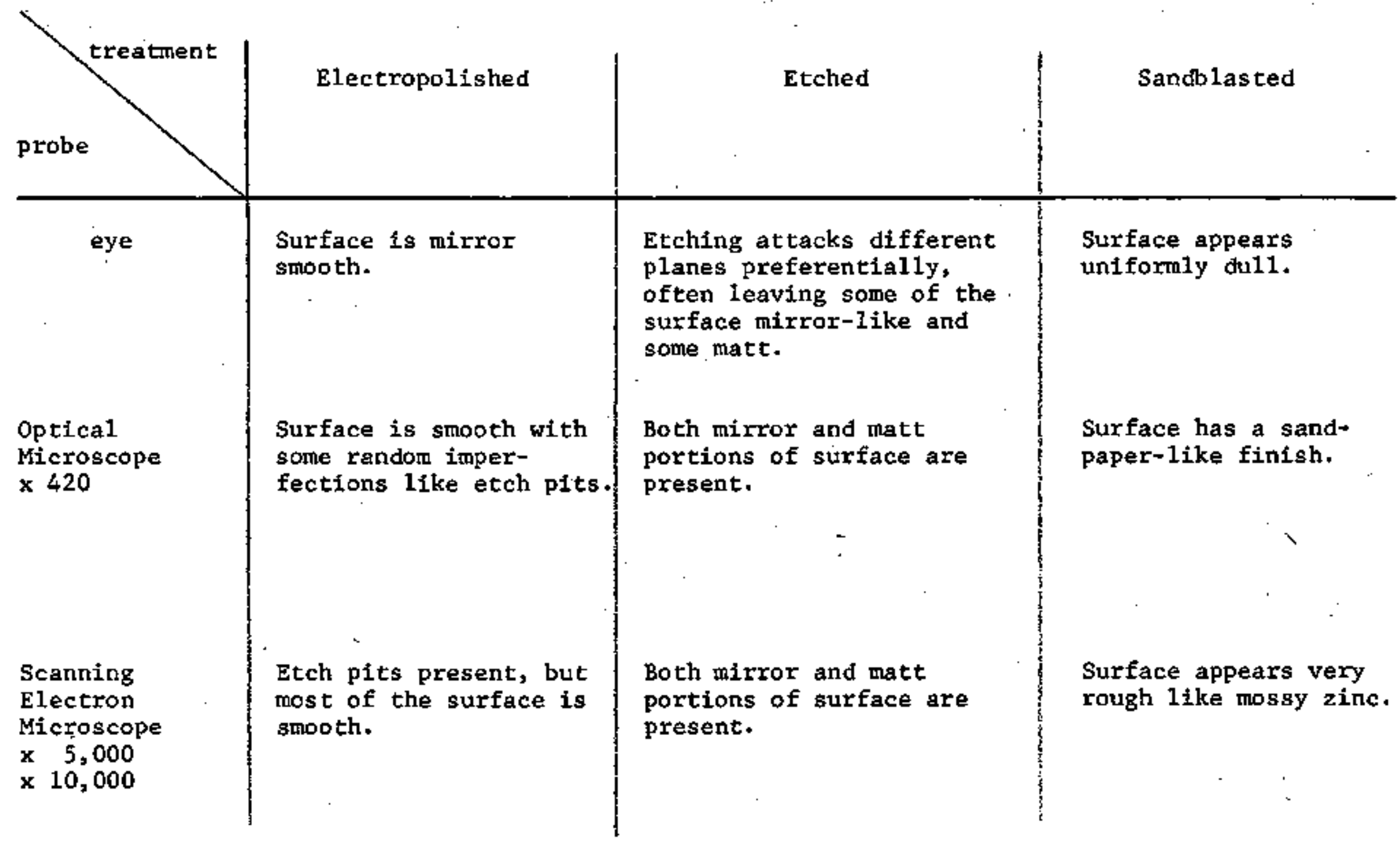




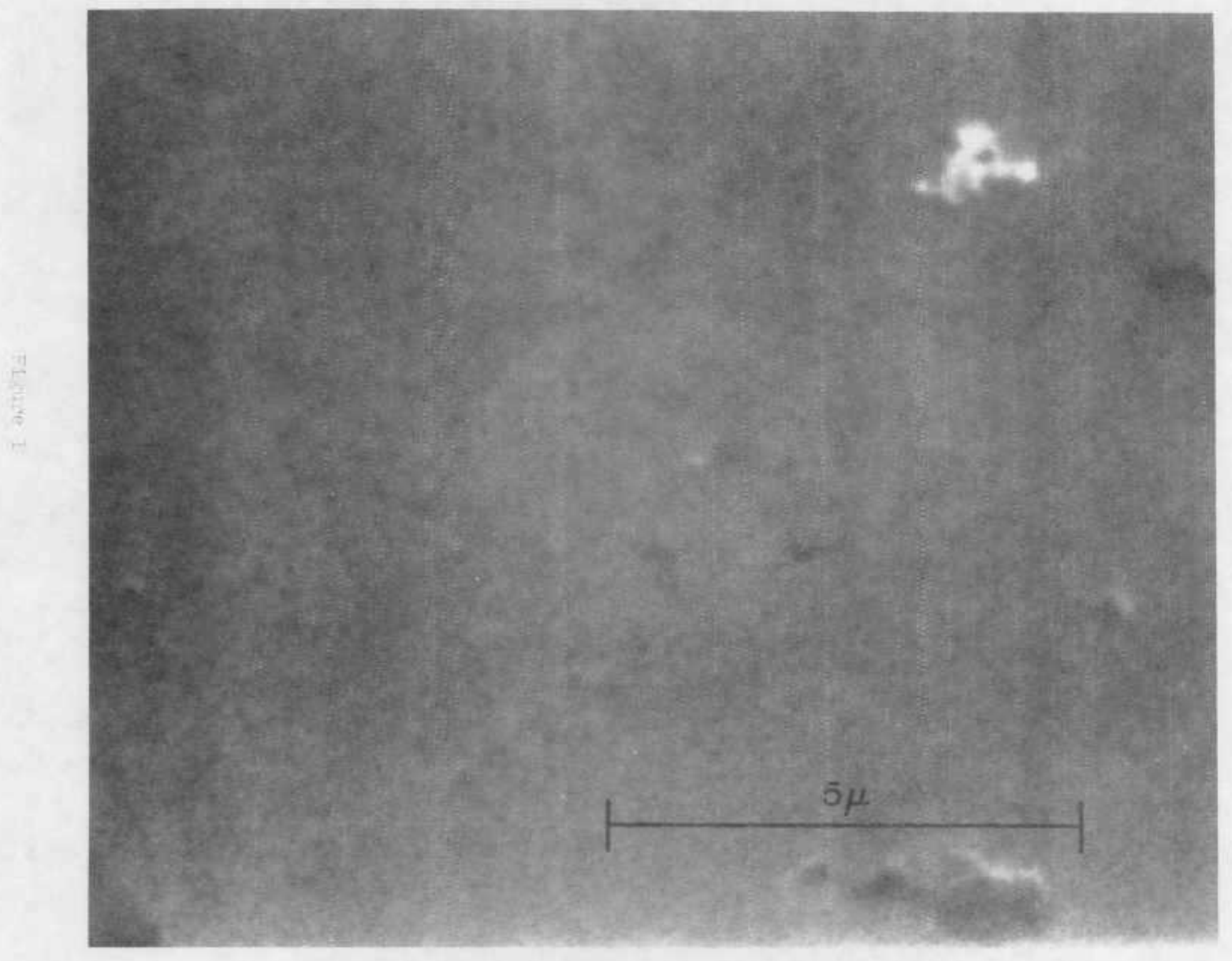




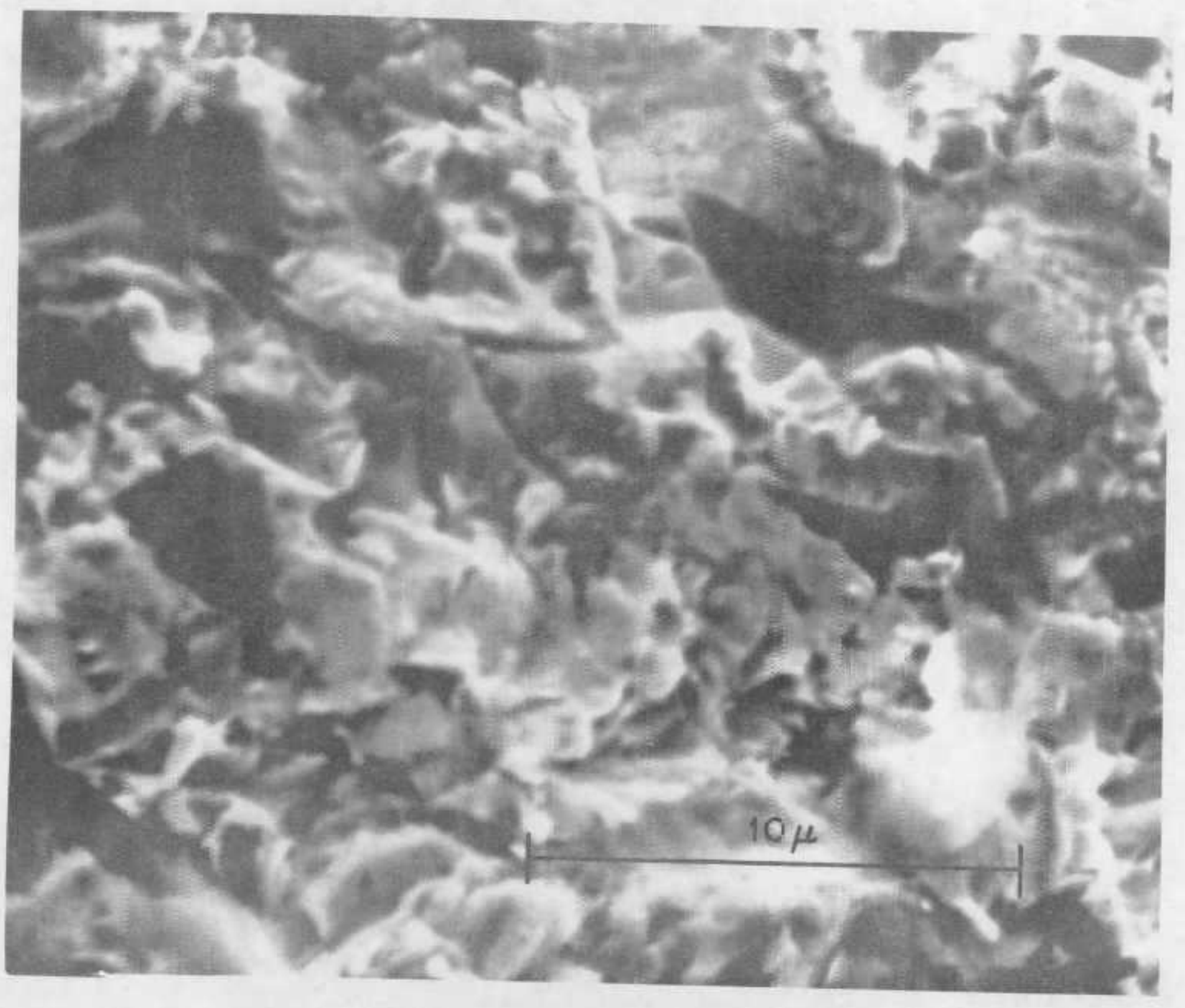




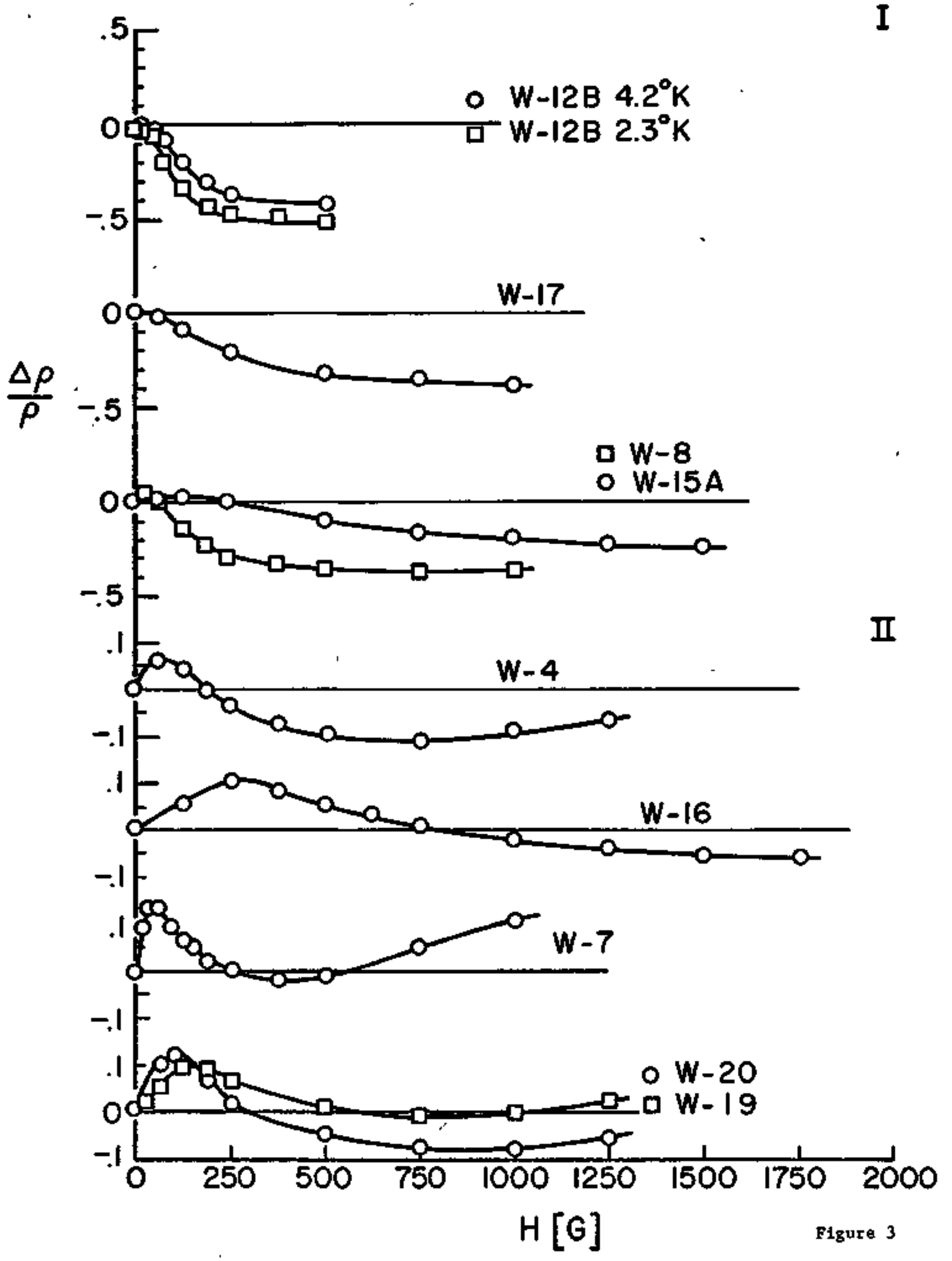




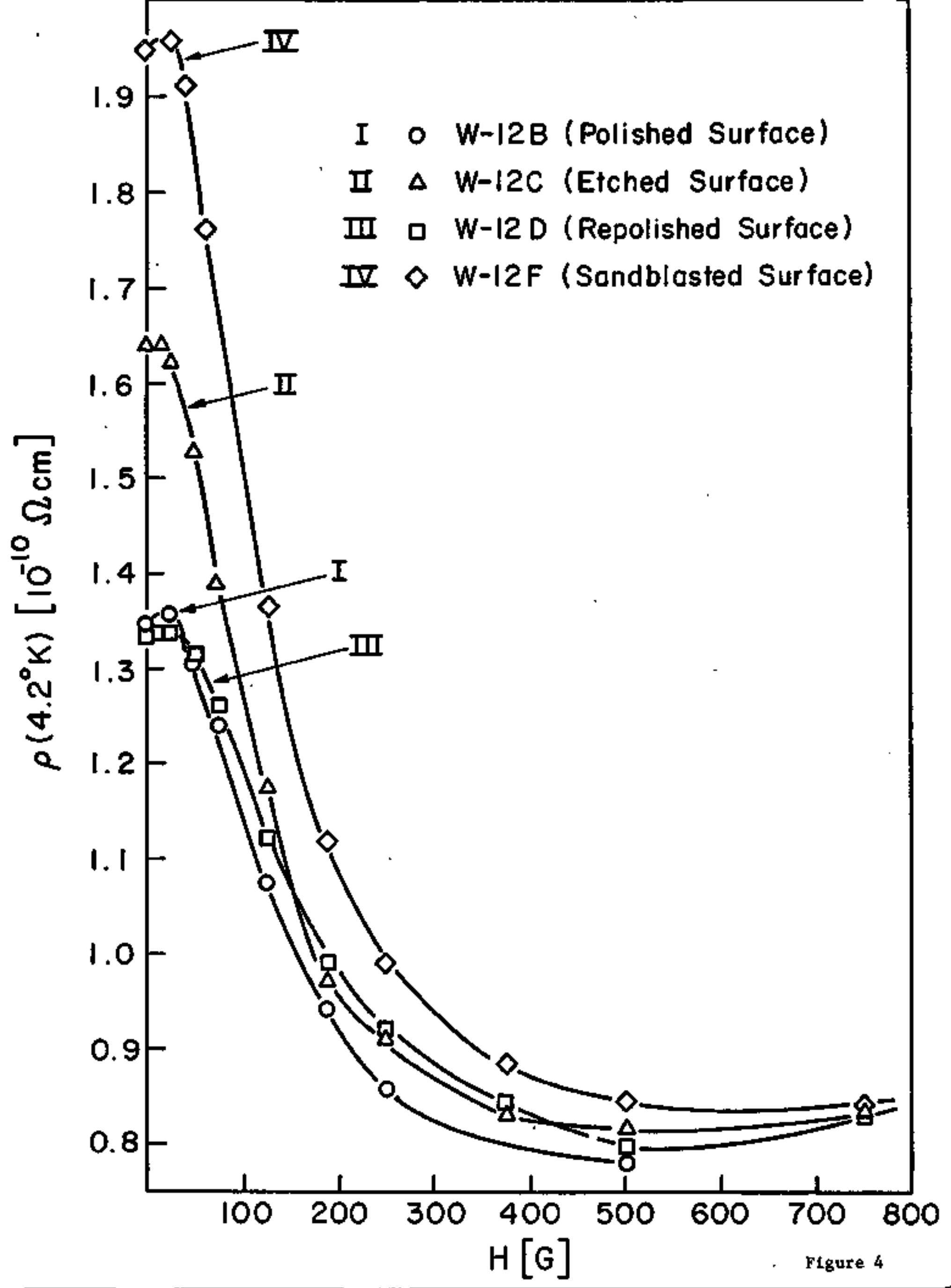




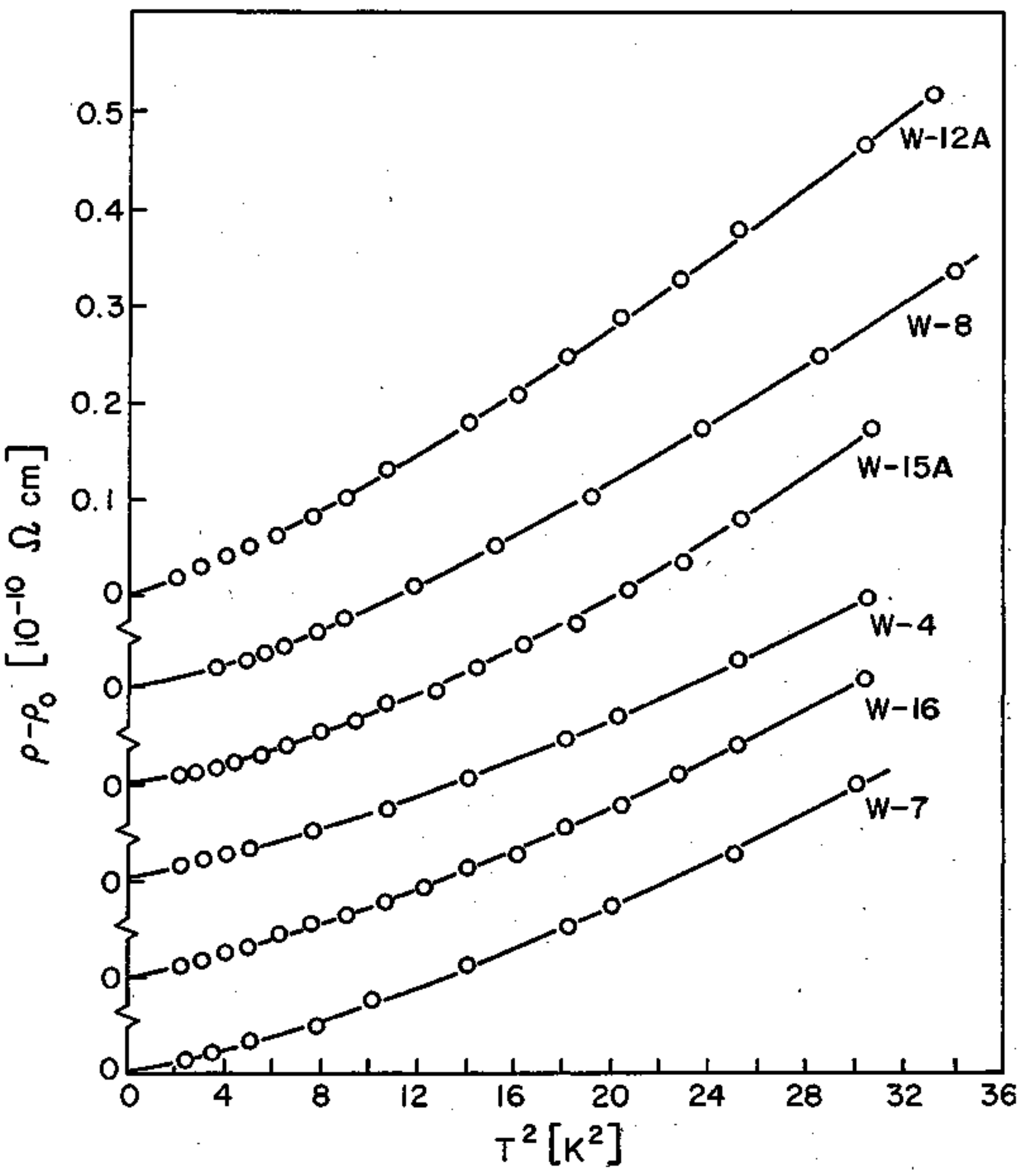






PIgure 6 


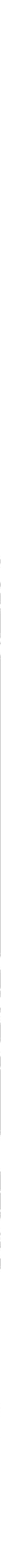




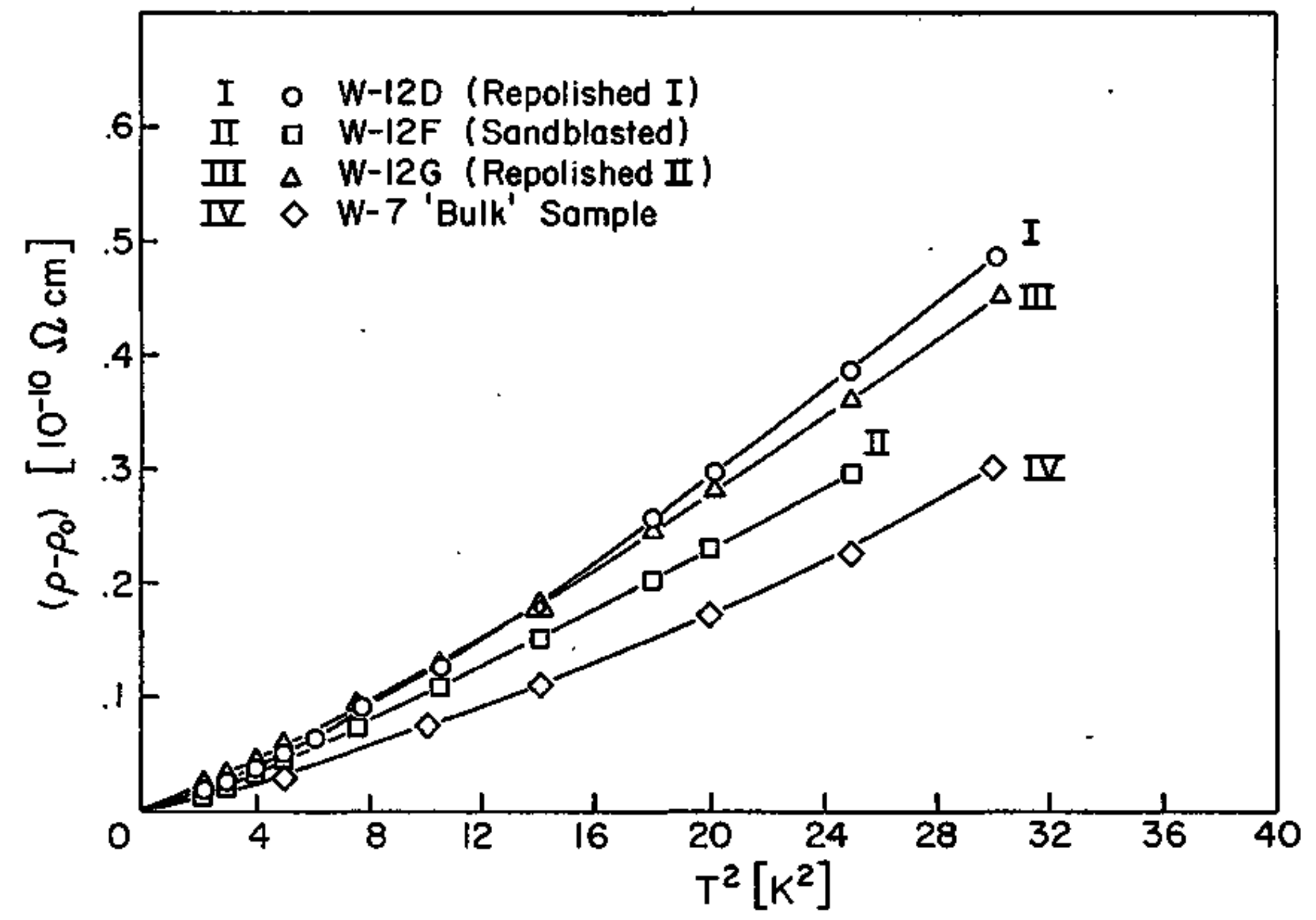

F1gure 9 


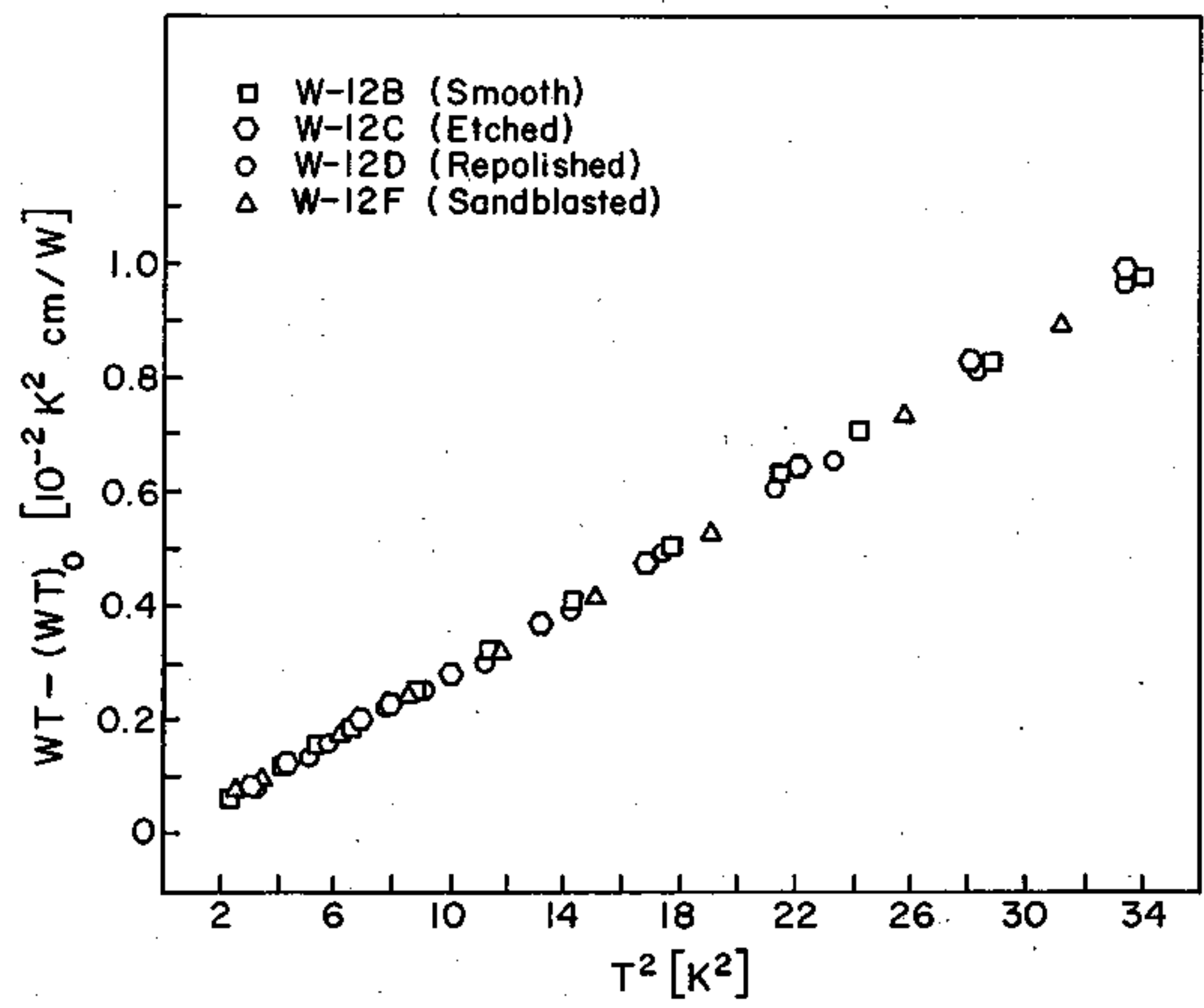

Figure 10 


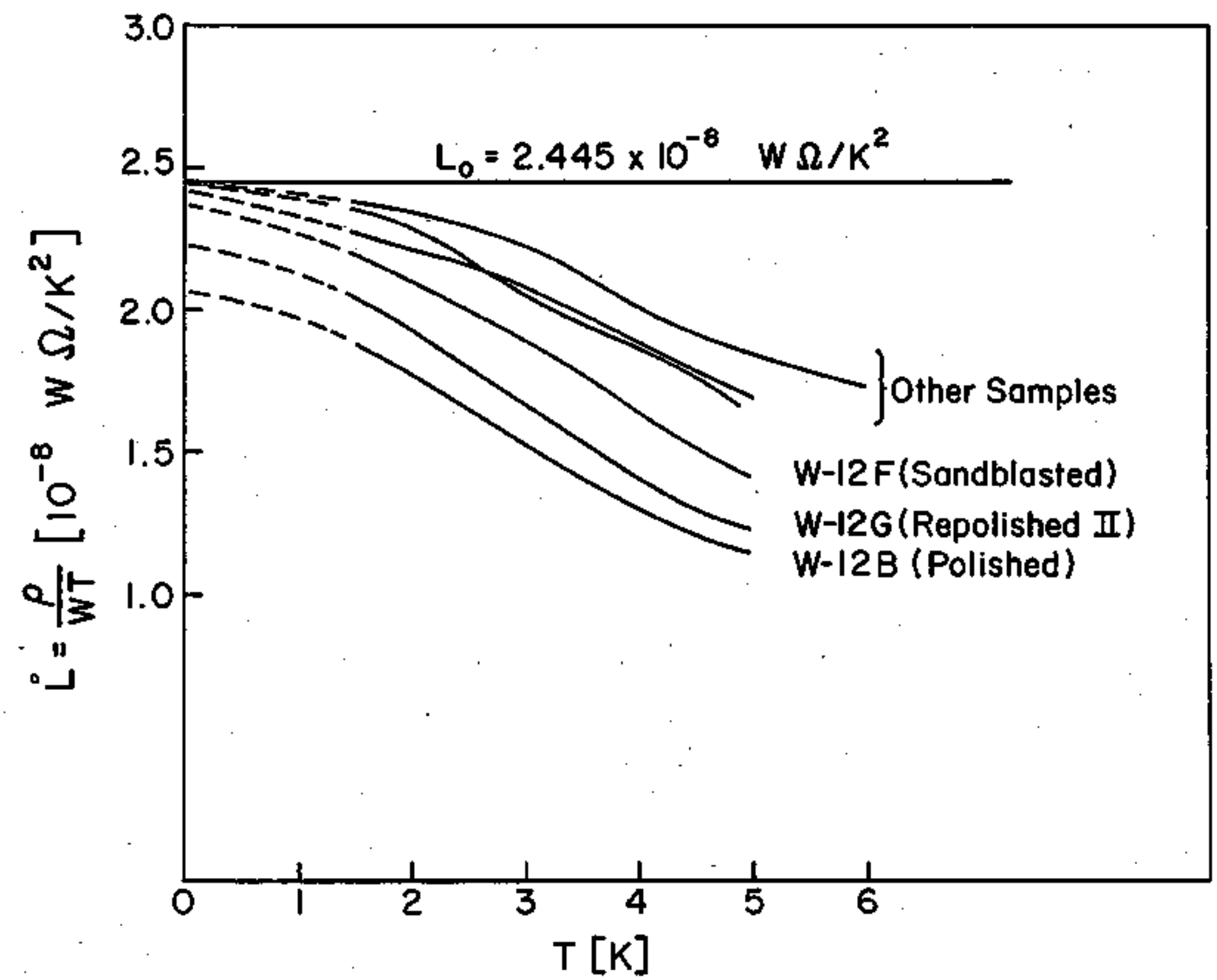

Pigure 11 
